https://doi.org/10.15407/ujpe64.7.613

T. NOSEK

for the NOvA Collaboration

Charles University, Faculty of Mathematics and Physics, Institute of Particle and Nuclear Physics

(2,V Holesovickach, 18000 Prague, Czech Republic; e-mail: tomas.nosek@mff.cuni.cz)

\title{
RESULTS ON NEUTRINO AND ANTINEUTRINO OSCILLATIONS FROM THE NOvA EXPERIMENT
}

\begin{abstract}
NOvA is a two-detector long-baseline neutrino oscillation experiment using Fermilab's $700 \mathrm{~kW}$ NuMI muon neutrino beam. With a total exposure of $8.85 \times 10^{20}+12.33 \times 10^{20}$ protons on target delivered to NuMI in the neutrino + antineutrino beam mode ( $78 \%$ more antineutrino data than in 2018), the experiment has made a $4.4 \sigma$-significant observation of the $\bar{\nu}_{e}$ appearance in $a \bar{\nu}_{\mu}$ beam, measured oscillation parameters $\left|\Delta m_{32}^{2}\right|, \sin ^{2} \theta_{23}$, and excluded most values near $\delta_{\mathrm{CP}}=\pi / 2$ for the inverted neutrino mass hierarchy by more than $3 \sigma$.

Keywords: neutrino oscillations, long-baseline experiment, NOvA, Fermilab.
\end{abstract}

\section{Introduction}

NOvA is a long-baseline neutrino oscillation experiment designed to make measurements of the muon neutrino $\left(\nu_{\mu}\right)$ disappearance and the electron neutrino $\left(\nu_{e}\right)$ appearance in Fermilab's NuMI (Neutrinos at the Main Injector) beam. Well tuned for the first oscillation maximum around a neutrino energy of $2 \mathrm{GeV}$ over $810 \mathrm{~km}$ baseline, the experiment studies primarily four channels of oscillations: $\nu_{\mu} \rightarrow \nu_{\mu}$ or $\nu_{\mu} \rightarrow \nu_{e}$ and $\bar{\nu}_{\mu} \rightarrow \bar{\nu}_{\mu}$ or $\bar{\nu}_{\mu} \rightarrow \bar{\nu}_{e}$. They allow us to address several concerns of neutrino oscillations:

1. mass ordering, i.e. normal $(\mathrm{NH})$ or inverted hierarchy $(\mathrm{IH})$ of neutrino mass eigenstates,

2 . direct $\mathrm{CP}$ violation $\left(\delta_{\mathrm{CP}}\right.$ phase) and

3. precise determination of $\theta_{23}$ and $\Delta m_{32}^{2}$ neutrino mixing parameters.

This paper reports the 2019 NOvA combined analysis of $8.85 \times 10^{20}$ POT (protons on target) neutrino data collected from Feb 2014 to Feb 2017 and 12.33× $10^{20}$ POT antineutrino data collected from Jun 2016 to Feb 2019 [1]. Neutrino oscillation parametrization, fits, predictions, and interpretation of the results were done within the standard oscillation model of 3 active neutrino flavors of electron, muon, and tau neutrinos $\left(\nu_{\tau}\right)[2]$.

\section{The NOvA Experiment}

The experiment consists of two large functionally identical detectors sitting $14.6 \mathrm{mrad}$ off the beam axis

(C) T. NOSEK, 2019

ISSN 2071-0194. Ukr. J. Phys. 2019. Vol. 64, No. 7
$810 \mathrm{~km}$ apart. This off-axis configuration reduces the uncertainty on energy of incoming neutrinos and suppresses the higher-energy neutrinos background producing neutral current interactions (NC) misidentified as $\nu_{e}$ charged current (CC). On the other hand, it also results in a lower intensity than in the on-axis region, mitigated by the size of the detectors and beam power upgrades.

The detectors are finely grained and highly active ( $\sim 65 \%$ active mass) liquid scintillator tracking calorimeters, which allow for a precise analysis of the neutrino interactions events. They are designed to be as similar as possible aside from the size: the Far Detector (FD) is $14 \mathrm{kt}$ and on the surface located in Ash River, Minnesota, the Near Detector (ND) is located underground in Fermilab, close enough to the neutrinos source to see a far greater flux with only $0.3 \mathrm{kt}$ of mass. Both are constructed out of extruded PVC cells $(3.9 \times 6.6 \mathrm{~cm}$ in cross-section and $15.5 / 3.8 \mathrm{~m}$ in length for FD/ND) filled with scintillator and equipped with a wavelength shifting fiber connected to an avalanche photodiode (APD). They collect light produced by charged particles subsequently amplified by APDs. The cells alternate in horizontal and vertical orientations to allow for a stereo readout. More information on detectors can be found in Ref. [3].

The NuMI beam is created following the decay of charged pions and kaons produced by $120 \mathrm{GeV}$ protons hitting a carbon target. These parent mesons are focused by two magnetic horns and decay in flight

613 


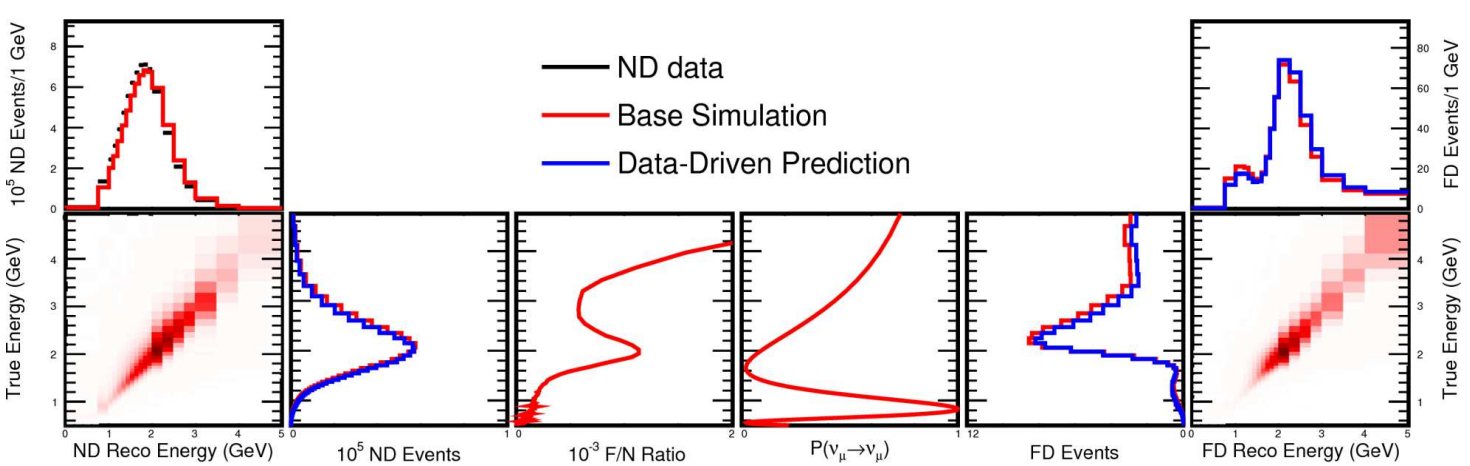

Fig. 1. Illustration of the NOvA's F/N technique. From left to right: reconstructed to true $\nu_{\mu}$ energy translation, $\mathrm{F} / \mathrm{N}$ ratio, $\nu_{\mu} \rightarrow \nu_{\mu}$ oscillation probability, true to reconstructed $\nu_{\mu}$ energy restoration. Base simulation in red (light), ND data-driven corrected prediction in blue (dark)

through the chain $K^{+}, \pi^{+} \rightarrow \mu^{+}+\nu_{\mu}$, with the muon then decaying as $\mu^{+} \rightarrow e^{+}+\nu_{e}+\bar{\nu}_{\mu}$. By switching the polarity of the horns, the opposite charge sign particles can be focused, thus effectively selecting an antineutrino beam. The resulting neutrino events sample composition in range $1-5 \mathrm{GeV}$ at ND is of $96 \% \nu_{\mu}$, $3 \% \bar{\nu}_{\mu}$ and $1 \% \nu_{e}+\bar{\nu}_{e}$ in the case of neutrino beam and $83 \% \bar{\nu}_{\mu}, 16 \% \nu_{\mu}$ and $1 \% \bar{\nu}_{e}+\nu_{e}$ in the case of antineutrino beam.

To identify and classify neutrino interactions, NOvA uses a method based on image recognition techniques known as Convolutional Visual Network $(\mathrm{CVN})$, see Ref. [4]. CVN treats every interaction in the detector as an image, with cells being pixels and collected charge being their color. When trained with simulated events and cosmic data, CVN can extract abstract topological features of neutrino-like interactions with convolutional filters (feature maps [4]). With an input of calibrated 2D pixelmap (two views of horizontal and vertical event projections), the output is a set of normalized classification scores ranging over the hypotheses of beam neutrinos event $\left(\nu_{\mu} \mathrm{CC}, \nu_{e} \mathrm{CC}, \nu_{\tau} \mathrm{CC}\right.$ and $\left.\mathrm{NC}\right)$, or cosmics. CVN has been used together with additional supporting PIDs: separate $\nu_{e}$ and $\nu_{\mu}$ cosmic rejection boosted decision trees and muon track identification in $\nu_{\mu}$ events.

NOvA's two identical detectors design enables us to employ data-driven predictions of FD observations. $\mathrm{FD} \nu_{\mu}$ and $\nu_{e}$ signal is predicted using ND $\nu_{\mu}$, whereas FD $\nu_{e}$ beam background is constrained using ND $\nu_{e}$ sample. This Far/Near (F/N) technique includes several steps (Fig. 1). First, the reconstructed neutrino energy spectrum is translated to the true energy using a simulated migration matrix. Second, the $\mathrm{F} / \mathrm{N}$ ratio

614 accounting for geometry, beam divergence, and detector acceptance is applied to create an unoscillated FD prediction. Then the FD spectrum is weighted by the oscillation probability for a given set of oscillation parameters. Finally, the true energy is smeared back again to the reconstructed energy via the migration matrix. As a reward, $\mathrm{F} / \mathrm{N}$ technique significantly reduces both neutrino flux and cross section systematic uncertainties. The ND reconstructed energy spectra of $\nu_{\mu}$ and $\bar{\nu}_{\mu}$ (the source of FD $\nu_{\mu}$ and $\nu_{e}$ signals) can be found in Fig. 2.

\section{Muon Neutrino and Antineutrino Disappearance}

The muon neutrino disappearance channel is primarily sensitive to $\left|\Delta m_{32}^{2}\right|$ and $\sin ^{2} 2 \theta_{23}$, and the precision with which they can be measured depends on the $\nu_{\mu}$ energy resolution. The energy of $\nu_{\mu}$ is reconstructed as a sum of the energy of a muon and the remaining hadronic energy. The former is estimated from the range of the muon track, the latter from the sum of the calibrated hits not associated with the track. To get the best effective use of the energy resolution, the data binning is optimized in two ways. First, the energy binning has finer bins near the disappearance maximum and coarser bins elsewhere. Second, the events in each energy bin are further divided into four populations, or "quartiles", of varying reconstructed hadronic energy fraction, which correspond to different $\nu_{\mu}$ energy resolutions. The divisions are chosen such that the quartiles are of equal size in the unoscillated FD simulation. The $\nu_{\mu}\left(\bar{\nu}_{\mu}\right)$ energy resolution is estimated to be $5.8 \%$ (5.5\%), $7.8 \%(6.8 \%), 9.9 \%(8.3 \%)$, and $11.7 \%$ (10.8\%) for

ISSN 2071-0194. Ukr. J. Phys. 2019. Vol. 64, No. 7 

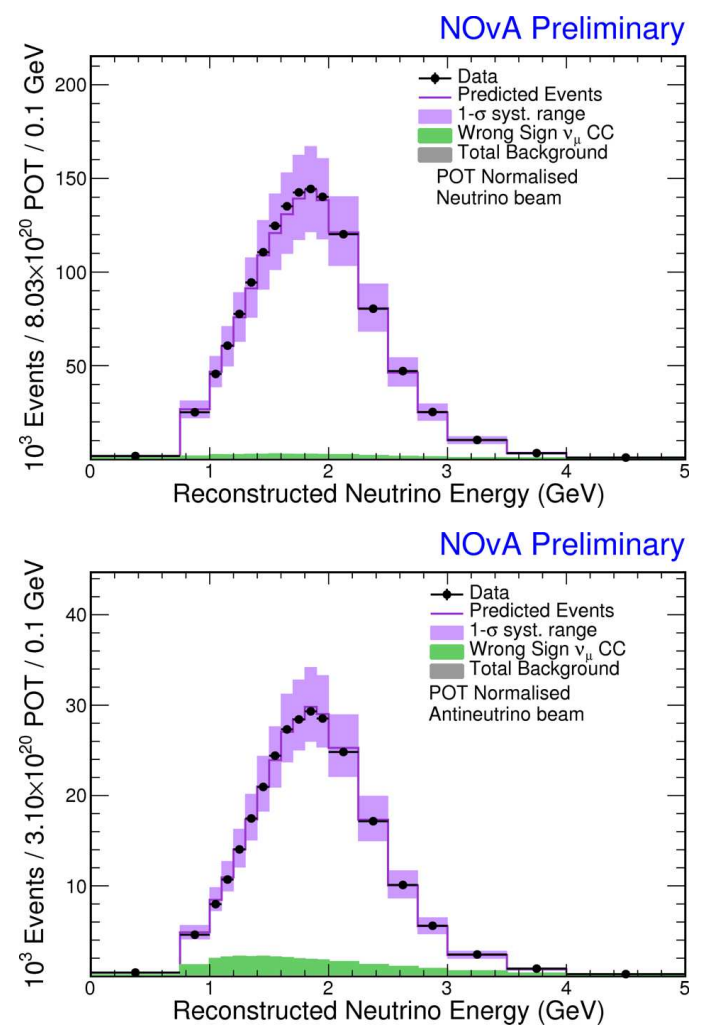

Fig. 2. ND selected $\nu_{\mu}$ (top) and $\bar{\nu}_{\mu}$ (bottom) reconstructed energies in data (black dots) and simulation (band). Each bin is normalized by its width

each quartile, ordered from lower to higher hadronic energy fraction. The $\mathrm{F} / \mathrm{N}$ technique is applied separately in quartiles, which has the additional advantage of isolating most of the cosmic and beam $\mathrm{NC}$ background events along with events of the worst energy resolution ( $4^{\text {th }}$ quartile).

The efficiency of the $\nu_{\mu}\left(\bar{\nu}_{\mu}\right)$ CC events selection is $31.2 \%(33.9 \%)$ with respect to true interactions in the fiducial volume and the purity $98.6 \%$ (98.8\%) in the FD samples. In total, there were 113 (102) $\nu_{\mu}$ $\left(\bar{\nu}_{\mu}\right) \mathrm{CC}$ candidates observed in FD with an estimated background of $4.2_{-0.6}^{+0.5}\left(2.2_{-0.4}^{+0.4}\right)$. FD data and the best fit prediction can be seen in Fig. 3 .

\section{Electron Neutrino and Antineutrino Appearance}

In order to maximize the statistical power of the $\nu_{e}$ selected events at FD, the sample is binned in both reconstructed energy and CVN score. There are two CVN bins of low and high purities (low and high
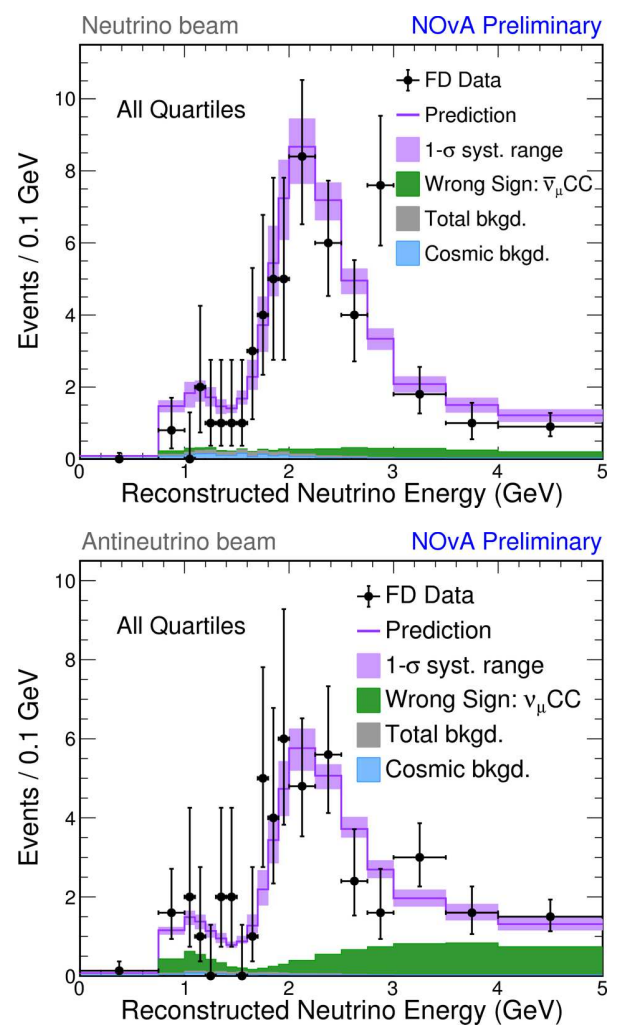

Fig. 3. FD data (black dots) selected $\nu_{\mu}$ (top) and $\bar{\nu}_{\mu}$ (bottom) candidates reconstructed energies compared to the best fit prediction (line) with $1 \sigma$ systematics uncertainty range. Summed over all quartiles of the hadronic energy fraction

PID), or "core" selection, and an additional "peripheral" bin. Events which fail the containment or cosmic rejection cuts, but do have a very high $\mathrm{CVN} \nu_{e}$ $\mathrm{CC}$ score, may be added to the peripheral sample. Because the events on the periphery are not always fully contained, they are summed into a single bin instead of estimating their energy (up to reconstructed 4.5 $\mathrm{GeV})$. The overall integrated selection efficiency of $\nu_{e}$ $\left(\bar{\nu}_{e}\right)$ is $62 \%(67 \%)$. The purity of the final predicted FD samples depends on the oscillation parameters, but ranges from $57 \%$ (55\%) to $78 \%(77 \%)$. The beam backgrounds are reduced by $95 \%$ (99\%).

To estimate FD beam backgrounds, the F/N technique is used with the ND $\nu_{e}$ sample. It consists of the beam $\nu_{e}$ and $\nu_{\mu} \mathrm{CC}$ or NC interactions misidentified as $\nu_{e}$ CC. Since each of these components oscillates differently along the way to the FD, the sample needs to be broken down into them. In the case of neutrino beam, the $\nu_{e}$ component is constrained by inspecting the low-energy and high-energy $\nu_{\mu} \mathrm{CC}$ spectra to 

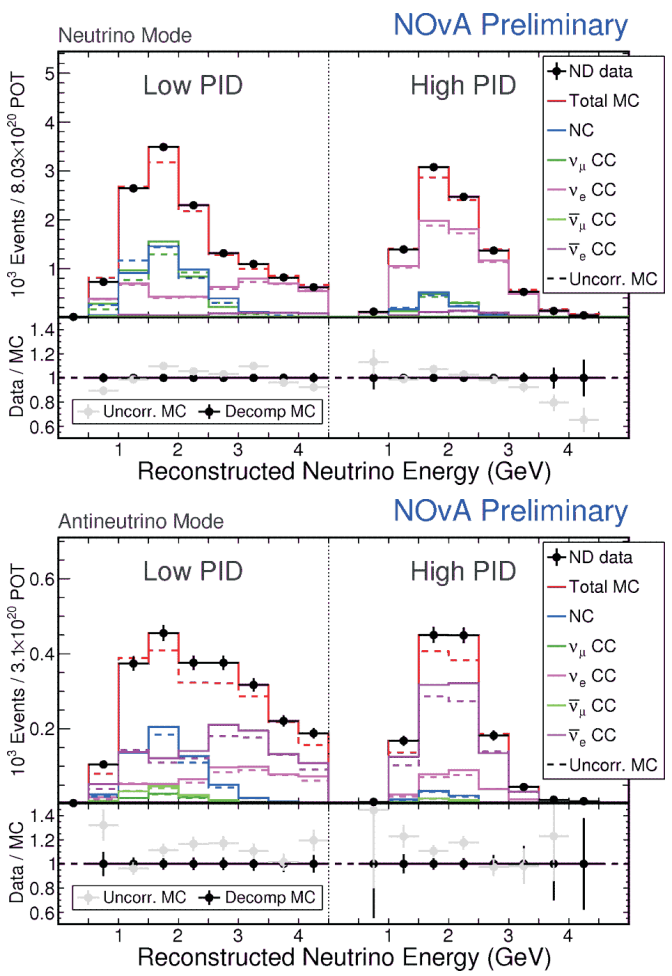

Fig. 4. ND selected $\nu_{e}$ (top) and $\bar{\nu}_{e}$ (bottom) reconstructed energy data (black dots), uncorrected simulation (dashed red) and data-driven correction (solid red). The selection is decomposed (broken down) into NC (blue), $\nu_{\mu} / \bar{\nu}_{\mu} \mathrm{CC}$ (dark/light green) and $\nu_{e} / \bar{\nu}_{e} \mathrm{CC}$ (light/dark magenta). Binned in two PID bins, which are correlated to lower and higher purities of $\nu_{e}+\bar{\nu}_{e}$

adjust the yields of the parent hadrons that decay into both $\nu_{\mu}$ and $\nu_{e}$ (track $\nu_{\mu}$ and $\nu_{e}$ to their common parents). The $\nu_{\mu}$ component is estimated from observed distribution of time-delayed electrons from the decay of stopped $\mu$. The rest is attributed to the $\mathrm{NC}$ interaction. In the case of antineutrino beam, the simulated components are evenly and proportionally scaled to match ND data in each bin. ND selections and their breakdowns, or "decomposition", can be seen in Fig. 4. The high PID bin is dominated by the beam $\nu_{e}+\bar{\nu}_{e}$, the low PID bin has a significant admixture of $\nu_{\mu}\left(\bar{\nu}_{\mu}\right) \mathrm{CC}$ and NC events. The beam background of the FD peripheral bin is estimated from the high PID bin of the core sample.

There were $58(27) \nu_{e}\left(\bar{\nu}_{e}\right)$ candidates in the FD data with the total expected background of $15.0_{-0.9}^{+0.8}\left(10.3_{-0.5}^{+0.6}\right)$ events of $7.0(5.3)$ beam $\nu_{e}+\bar{\nu}_{e}$, $0.7(0.2) \nu_{\mu}+\bar{\nu}_{\mu}, 3.1$ (1.2) NC events, 3.3 (1.1) cosmic-
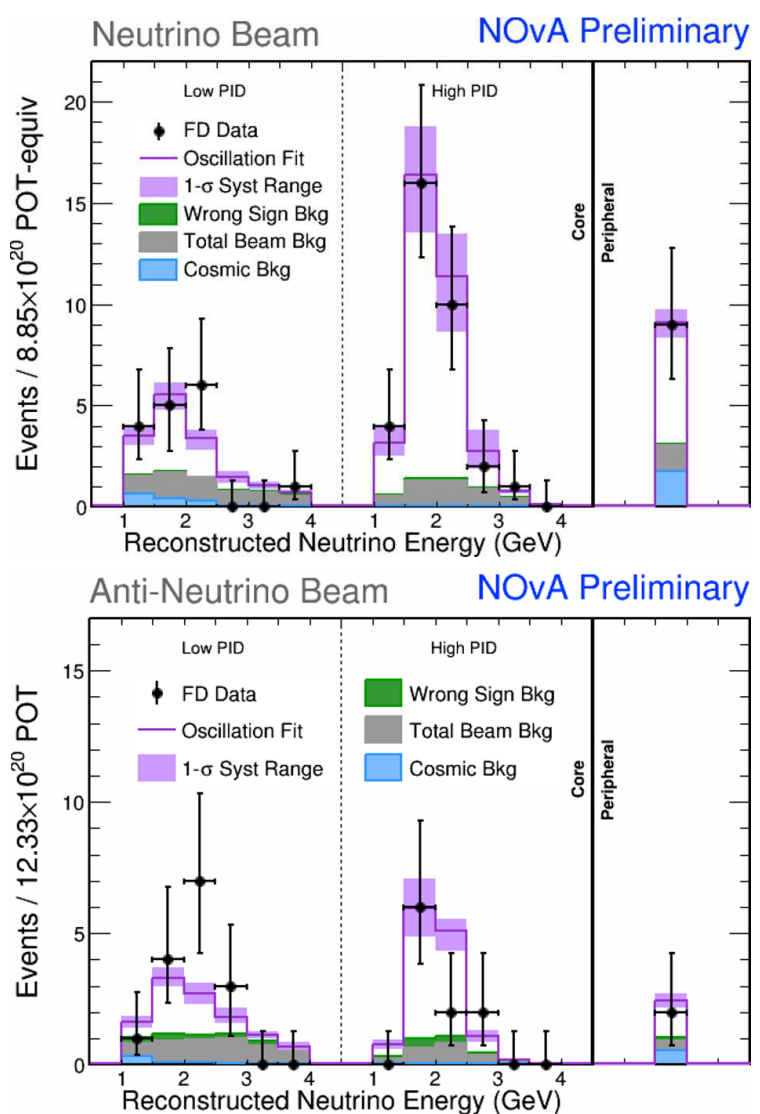

Fig. 5. FD data (black dots) selected $\nu_{e}$ (top) and $\bar{\nu}_{e}$ (bottom) candidates reconstructed energies binned in low and high PID bins and peripheral sample with energies up to $4.5 \mathrm{GeV}$. The best fit prediction (purple band) shows the expected background of wrong sign (green), other beam backgrounds (grey) and cosmics (blue) as shaded areas

ray-induced events, $0.4(0.3)$ others and $0.6 \bar{\nu}_{e}\left(2.2 \nu_{e}\right)$ from the wrong sign component of the $\nu_{\mu}\left(\bar{\nu}_{\mu}\right)$ sample. The FD data and the best fit predictions can be seen in Fig. 5. The antineutrino data give a $4.4 \sigma \mathrm{ev}-$ idence of the $\bar{\nu}_{e}$ appearance in $\bar{\nu}_{\mu}$ beam (an excess over predicted background).

\section{Constraints on Oscillation Parameters}

To obtain oscillation parameters, a simultaneous fit of joint $\nu_{e}+\nu_{\mu}$ and both the neutrino and antineutrino data was performed. Systematic uncertainties are incorporated as nuisance parameters with Gaussian penalty term, appropriately correlated between all the data sets. The leading systematics are worth a note: detector calibration (calorimetric energy scale), 
light production and collection model and muon energy scale (abs. + rel.) for the $\nu_{\mu}$ disappearance; detector response and calibration, neutrino crosssections and actual ND to FD differences for the $\nu_{e}$ appearance. Several oscillation parameters are taken as inputs from other measurements: solar parameters $\theta_{12}$ and $\Delta m_{12}^{2}$, the mixing angle $\theta_{13}$ and its uncertainty were taken from reactor experiments, all in Ref. [2]. The best fit is

$$
\begin{aligned}
& \Delta m_{32}^{2}=2.48_{-0.06}^{+0.11} \times 10^{-3} \mathrm{eV}^{2}, \\
& \sin ^{2} \theta_{23}=0.56_{-0.03}^{+0.04}, \\
& \delta_{\mathrm{CP}} / \pi=0.0_{-0.4}^{+1.3},
\end{aligned}
$$

which corresponds to $\mathrm{NH}$ and the uppper $\theta_{23}$ octant $\left(\mathrm{UO}, \theta_{23}>45^{\circ}\right.$ ). All confidence levels (C.L.) and contours are constructed following the Feldman-Cousins approach [7].

The $90 \%$ C.L. allowed region for a combination of $\Delta m_{32}^{2}$ versus $\sin ^{2} \theta_{23}$ in the $\Delta m_{32}^{2}>0$ halfplane, together with other results from MINOS (2014) [8], T2K (2018) [9], IceCube (2018) [10] and Super-Kamiokande (2018) [11] overlaid is shown in Fig. 5. There is a clear consistency within all experiments despite that NOvA data asymmetrically point to UO and disfavor lower $\theta_{23}$ octant $\left(\mathrm{LO}, \sin ^{2}<0.5\right)$ at about $1.6 \sigma$ C.L.

Fig. 7 shows the 1, 2 and $3 \sigma$ C.L. allowed regions for $\sin ^{2} \theta_{23}$ versus $\delta_{\mathrm{CP}}$ in both cases of $\mathrm{NH}$ and $\mathrm{IH}$ (mass ordering). It is worth noticing that the values of $\delta_{\mathrm{CP}}$ around $\pi / 2$ are excluded at $>3 \sigma$ C.L. for IH, similarly to the previous NOvA neutrino only analysis [5]. On the other hand, rather weak constraints on $\delta_{\mathrm{CP}}$ itself allow all possible values $[0,2 \pi]$ for the case of $\mathrm{NH}$ and UO. NH is preferred with $1.9 \sigma$ significance.

\section{Future Prospects}

NOvA is expected to run till 2025 with about an equal total exposure of neutrino and antineutrino beams. Moreover, several accelerator upgrades to enhance the beam performance are planned for the next years. Based on these prerequisities and projected 2019 analysis techniques, there is a possibility of more than $3 \sigma$ sensitivity to hierarchy resolution for $30-50 \%$ of all possible $\delta_{\mathrm{CP}}$ (up to $5 \sigma$ for favorable true values of oscillation parameters: $\mathrm{NH}$ and $\left.\delta_{\mathrm{CP}}=3 \pi / 2\right)$. In addition, more than $2 \sigma$ sensitivity to $\mathrm{CP}$ violation in the case of $\delta_{\mathrm{CP}}=\pi / 2$ or $3 \pi / 2$ (maximal violation) is expected.

ISSN 2071-0194. Ukr. J. Phys. 2019. Vol. 64, No. 7

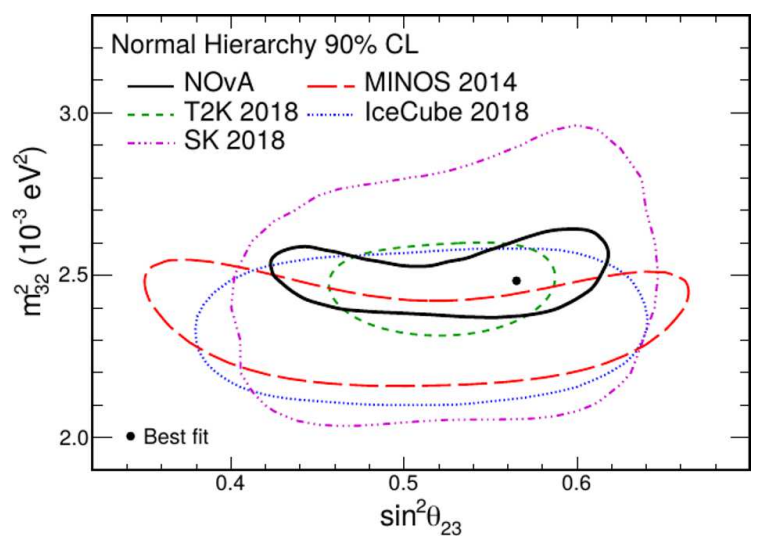

Fig. 6. Comparison of the allowed regions of $\Delta m_{32}^{2}$ vs. $\sin ^{2} \theta_{23}$ parameter space at the $90 \%$ confidence level as obtained by recent experiments

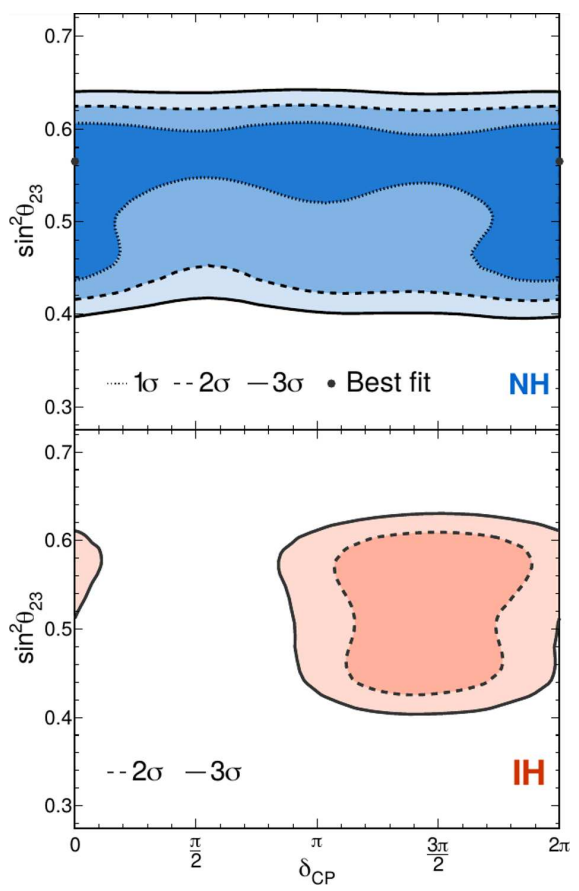

Fig. 7. 1,2 , and $3 \sigma$ allowed regions of $\sin ^{2} \theta_{23}$ vs. $\delta_{\mathrm{CP}}$ neutrino oscillation parameter space consistent with the $\nu_{e}$ appearance and $\nu_{\mu}$ disappearance data. The top panel corresponds to the case of normal hierarchy $(\mathrm{NH})$ of neutrino masses $\left(\Delta m_{32}^{2}>0\right)$, the bottom one to the inverted hierarchy (IH, $\left.\Delta m_{32}^{2}<0\right)$

To further improve the neutrino oscillation analysis and to extend the reach of the experiment, NOvA started an intensive test beam program in early 2019. This should focus on the simulation tun- 
ing, systematics study and their reduction, validation and training of the reconstruction or machine learning algorithms.

\section{Conclusions}

New antineutrino data from NOvA $\left(12.33 \times 10^{20}\right.$ POT in total) has been analyzed together with existing neutrino data $\left(8.85 \times 10^{20} \mathrm{POT}\right)$. The measurements are well consistent with the standard oscillation model of 3 active neutrino flavors. NOvA observes $4.4 \sigma$ evidence for the $\bar{\nu}_{e}$ appearance in $\bar{\nu}_{\mu}$ beam. The results of joint analysis of neutrino and antineutrino and both $\nu_{\mu}$ disappearance and $\nu_{e}$ appearance channels give the parameters estimates of $\sin ^{2} \theta_{23}=$ $=0.56_{-0.03}^{+0.04}$ and $\Delta m_{32}^{2}=2.48_{-0.06}^{+0.11} \times 10^{-3} \mathrm{eV}^{2}$, which are in a good agreement with other accelerator and atmospheric oscillation experiments. The data prefer $\theta_{23}$ upper octant at $1.6 \sigma$ and the normal hierarchy of neutrino masses at $1.9 \sigma$ and also disfavor the inverted hierarchy for $\delta_{\mathrm{CP}}$ around $3 \pi / 2$ at more than $3 \sigma$. NOvA plans to continue running till 2025 in both neutrino and antineutrino beam modes.

I would like to thank the organizers for an inspiring NTIHEP2019 conference. This work was supported by MSMT CR (Ministry of Education, Youth and Sports, Czech Republic).

1. M.A. Acero et al.. First measurement of neutrino oscillation parameters using neutrinos and antineutrinos by NOvA. FERMILAB-PUB-19-272-ND, arXiv:1906.04907 [hep-ex].

2. M. Tanabashi et al. [Particle Data Group]. Review of Particle Physics. Phys. Rev. D 98, 030001 (2018).

3. D.S. Ayres et al. [NOvA Collaboration]. The NOvA technical design report. FERMILAB-DESIGN-2007-01.

4. A. Aurisano et al. A convolutional neural network neutrino event classifier. JINST 11, P09001 (2016), arXiv:1604.01444 [hep-ex].

5. P. Adamson et al. [NOvA Collaboration]. New constraints on oscillation parameters from $\nu_{e}$ appearance and $\nu_{\mu}$ dis- appearance in the NOvA experiment. Phys. Rev. D 98 032012 (2018), arXiv:1806.00096 [hep-ex].

6. P. Adamson et al. [MINOS Collaboration], The NuMI neutrino beam. Nucl. Instrum. Meth. A 806, 279 (2016), arXiv:1507.06690 [physics.acc-ph].

7. G.J. Feldman, R.D. Cousins. A unified approach to the classical statistical analysis of small signals. Phys. Rev. D 57, 3873 (1998), arXiv:physics/9711021 [physics.data-an].

8. P. Adamson et al. [MINOS Collaboration]. Combined analysis of $\nu_{\mu}$ disappearance and $\nu_{\mu} \rightarrow \nu_{e}$ appearance in MINOS using accelerator and atmospheric neutrinos. Phys. Rev. Lett. 112, arXiv:1403.0867 [hep-ex].

9. K. Abe et al. [T2K Collaboration]. Search for CP violation in neutrino and antineutrino oscillations by the $\mathrm{T} 2 \mathrm{~K}$ experiment with $2.2 \times 10^{21}$ protons on target. Phys. Rev. Lett. 121, 171802 (2018), arXiv:1807.07891 [hep-ex].

10. M.G. Aartsen et al. [IceCube Collaboration]. Measurement of atmospheric neutrino oscillations at $656 \mathrm{GeV}$ with ice cube deep core. Phys. Rev. Lett. 120, 071801 (2018), arXiv:1707.07081 [hep-ex].

11. K. Abe et al. [Super-Kamiokande Collaboration]. Atmospheric neutrino oscillation analysis with external constraints in Super-Kamiokande I-IV. Phys. Rev. D 97, 072001 (2018), arXiv:1710.09126 [hep-ex].

Received 08.07.18

T. Носек, від Колаборацї NOvA

ОСЦИЛЯЦЇ̈ НЕЙТРИНО

ТА АНТИНЕЙТРИНО. ЕКСПЕРИМЕНТ NOvА

$\mathrm{P}$ е $з$ ю м е

NOvA - експеримент 3 двома детекторами 3 подовженою базою для вимірювання нейтринних осциляцій за допомогою струменя мюонних нейтрино на $700 \mathrm{~kW}$ NuMi. 3 протонним струменем, спрямованим на мішень NuMi iз загальною експозицією $8.85 \times 10^{20}+12 / 33 \times 10^{20}$, в режимі нейтрино + антинейтрино (на $78 \%$ процентів більше антинейтрино, ніж у 2018 році), експеримент досяг достовірності $4.4 \sigma$ появи $\bar{\nu}_{e}$ в пучку $\bar{\nu}_{\mu}$, було виміряно параметри осциляції $\left|\Delta m_{32}^{2}\right|, \sin ^{2} \theta_{23}$, а також було виключено більшість значень, близьких до $\delta_{\mathrm{CP}}=\pi / 2$ для зворотних нейтрино, більш ніж на $3 \sigma$. 